\title{
Expanding the Use of Orthodontic Appliance in the Management of Maxillo-Mandibular Fractures
}

\author{
MANAR KHATER, M.Sc.; AYMAN NOAMAN EL-HENAWY, M.D.; AHMED FATHY ABO RADY, M.D.; \\ ASHRAF EL-SEBAEI MOHAMMED, M.D. and AHMED SAFWAT SALEM, M.D.
}

The Department of General Surgery, Plastic Surgery Unit, Faculty of Medicine, Cairo University

\begin{abstract}
Background: Maxillofacial fractures are common presentation in Plastic Surgery Emergency Department. Many techniques of maxillary mandibular fixation have evolved over the years. Orthodontic appliance technique as a reliable method for Maxillary-mandibular fixation has been introduced.

Methods: Prospective clinical study was conducted on 20 patients complaining of different types of maxillo-facial fractures who were candidates for maxillary-mandibular fixation with or without open reduction and internal fixation. The objective of the study is to demonstrate technical feasibility, overall outcomes and complications of the technique, in the period from October 2017 till March 2018 at in the Emergency Plastic Surgery Department in Cairo University Hospital.

Results: In suitable cases orthodontic brackets and elastics can be used as an effective method of Maxillary-mandibular fixation with good patients' satisfaction. It can reduce the hospital admission rate and need for general anesthesia; it will not only reduce costs for the hospital but also costs for the patient.

Conclusion: Orthodontic brackets technique's stability allowed using it as a maxillary mandibular fixation even alone or with open reduction and internal fixation, thus it used as an effective method for maxillary-mandibular fixation, and has proven to cause a significant impact on returning the patients to their pre-traumatic bite. This makes it able to replace the Erich arch bar in maxillofacial fracture as a method of maxillary-mandibular fixation. Orthodontic brackets technique has shown to offer patients better oral hygiene, convenience and no mucosal injuries.
\end{abstract}

Key Words: Maxillofacial fractures - Maxillary-mandibular fixation - Orthodontic appliance.

\section{INTRODUCTION}

Maxillofacial trauma can be complex in nature and can be part of the multiple trauma victims. The incidence is more common among males with a ratio of nearly 4:1 over female [1]. Maxillofacial fractures are due to fall from height, direct blow, motor vehicle accidents, sport injuries or falls [2]. After proper reduction of any maxillo-facial fracture immobilization of the fracture site must be done adequately with or without internal fixation to be sure that proper healing occurs [3].

Simple mandibular fractures can be managed only using maxillary-mandibular fixation if there is adequate number of teeth and if the fracture site is on the tooth bearing part. Healing can be achieved within 4 weeks and maxillary-mandibular fixation can be done without anesthesia. The most temporary method used to maintain proper occlusion is the maxillary-mandibular fixation. There is variation of methods of maxillary-mandibular fixation used as MMF screws, arch bar, interdentally wiring and the modernly era of orthodontic appliance [4].

In all the previously mentioned procedures used for IMF from the wiring techniques to the most commonly and popularly used (Erich arch bar) and the modernly era of orthodontic appliances they all aim to provide good immobilization for fixed fractures in a normal functional occlusion between the mandible and maxillary teeth. This method has many advantages as it can be used without anesthesia, no need for wide mouth opening during application, no gingival or mucosal injury, application and removal can be done without anesthesia, better oral hygiene and patient convenience, costs for both the hospital and the patient can be reduced by minimizing the rate of patient admission and the need of general anesthesia [5].

\section{METHODOLOGY}

This prospective clinical study was conducted on 20 patients complaining of multiple maxillofacial fractures who were candidates for maxillarymandibular fixation with or without open reduction and internal fixation, in the period from October 2017 till September 2018 at the Emergency Plastic 
Surgery Department in Kasr Al-Ainy Hospital Cairo University.

After primary survey has been done, treatment started with evaluating the injury, taking an accurate history and mode of trauma. Full examination of the midface, the mandible and cervical spines should be done precisely, laboratory investigations, photographic evaluation and documentation.

Pre-operative photographs were taken in standing position from lateral, frontal, oblique views and intra oral anterior, 2 laterals and occlusal upper and lower views. Post-operative photographs were also taken with orthodontic appliance and after removal in the same views to compare the preoperative photos.

All patients underwent panoramic view radiographs (Panorex), and CT scans for the facial bones including axial and coronal cuts with $3 \mathrm{D}$ reconstruction. Post-operatively all patients were followed-up with panoramic view radiographs (Panorex) after 2-3 days and 1, 3, 6 months postoperatively and a CT Scan was done to patients with multiple or comminuted maxillofacial fractures and if the fracture sites is not clear in panoramic view in simple fractures.

All patients in the study were subjected to MMF using the orthodontic brackets in the same day of the admission to the hospital after completion of their primary survey and assessment. Open reduction and internal fixation was done within 2-3 days after in indicated patients. 8 patients underwent maxillary-mandibular fixation only using orthodontic appliance bedside without anesthesia resulting a sufficient reduction and good occlusion and they didn't need ORIF. 12 patients underwent ORIF after the maxillary-mandibular fixation with orthodontic brackets (10 patients underwent the maxillamandibular fixation bedside without anesthesia before scheduling them for surgery for internal fixation and 2 patients underwent maxillarymandibular fixation using orthodontic appliance intra-operatively under general anesthesia due to the presence of other concomitant injuries that interfered with that application of the orthodontic brackets bedside).

Patients were placed in supine position with the head fitting in a suitable sized head rest with the neck slightly extended. All patients were subjected to standard maxillo-mandibular fixation procedures. Our target for treatment was to return to the pre-traumatic occlusion state and restore the normal function. A Good view of the mouth can be achieved by using a cheek retractor. The teeth are thoroughly cleaned with polishing paste using a polishing brush then rinsed with water and dried using cotton rolls. [Tooth must be cleaned from any food debris and polished from any stains before application of brackets].

Bonding by acid etch technique: Put acid etch (the blue gel) in the middle third of the tooth to make micro irregularities and pores in the tooth surface so the smooth surface of the tooth will transform into rough surface. Then tooth rinsed with water and dried carefully using cotton rolls. Bonding agent [liquid adhesive material seep in between the micro-irregularities of the etched tooth surface] placed on the etched enamel surface of the tooth, then dental light curing (LED light emitting diode curing light) is used for hardening and curing of bonding agent.

Composite [adhesive material that plays an important role for adherence of orthodontic brackets] is placed on the bracket. The brackets are cemented to the tooth with composite. Removing excess composite resin around the bracket, the brackets are protected from contact with Saliva for several minutes. Then the composite is cured with light cure [used for hardening and curing of the composite]. Produce a bracket for each tooth by the same technique. Incorrect bracket positioning will lead to incorrect tooth position and ultimately affect the function result.
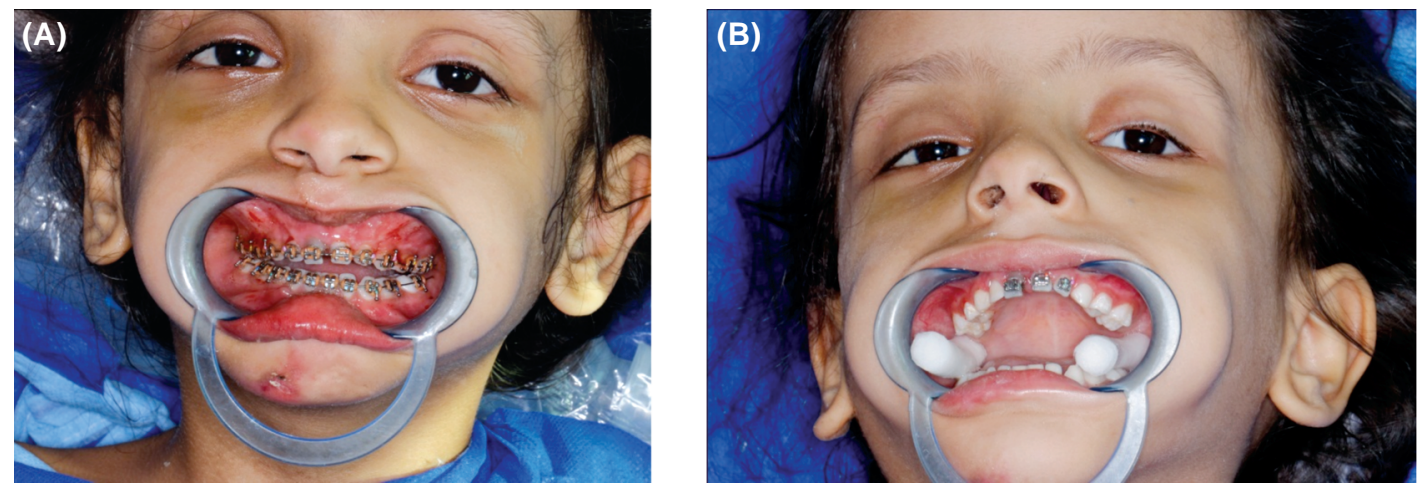

Fig. (1A,B): A 6y old female patient during the application of the orthodontic brackets without anesthesia and fully awake. 
A nickel titanium arch wire is used to achieve alignment and secured into the bracket slot by rubber bands. Arch wire should be cut accurately to the length of the dental arch. Accuracy in this regard will prevent injury to the adjacent soft tissues by protruding ends.

Maxillo-mandibular fixation is done by placing ligature wires around the upper and lower bracket hooks, twisting the two ends of wire together; it is always done in a clockwise manner, so that later on removal of wires can be done in anticlockwise manner. Then the elastics can be placed around the bracket hooks. Elastics and wire traction will reduce the fracture and bring it into the normal occlusion. The brackets can be removed by grasping them with a clamp and exerting with a sharp rotational force.

The decision of either managing the patient using only MMF or the need for ORIF after applying the orthodontic brackets was taken according to site and type of the fracture. Duration of the MMF was between 2 to 6 weeks in case of MMF alone and between 2 to 3 weeks in case of MMF with ORIF. All orthodontic brackets were removed at the $6^{\text {th }}$ week as an outpatient procedure without anaesthesia.

All patients were discharged within 24-72 hours after their operation. They were prescribed oral antimicrobial regimen and pain killers in addition to mouthwash. They were instructed to follow a soft diet. Post-operative visits were scheduled 1, 6,12 and 24 weeks post-operative.

Assessment of occlusion clinically and radiologically, assessment of application timing and assessment of patients' satisfaction, hygiene and convenience to the procedure were done to all patients.

A survey with rating scale questions was given to all patients of the study after removal the orthodontic appliances in which the patients had to give a rate from 1-10 for the following items:

1- Convenience during application and removal of the orthodontic appliance.

2- Oral hygiene during the time of application of the orthodontic appliance.

3- Convenience with oral feeding during the time of application of the orthodontic appliance.

4- The presence of mucosal injuries with or without pain during this period.

\section{RESULTS}

This study was performed on 20 patients who were complaining of maxillofacial fractures and candidates for maxillary-mandibular fixation who were managed by orthodontic appliance. The age range in the study varied from 5 to 60 years (mean age: 20.5$)$.

A total of 43 fractures were presented in the twenty patients they included parasymphseal, body, angle, condyle, subcondyle, zygomatico-maxillary complex fractures.

Fig. (2): Number of various types of fractures in all twenty patients of the study.

\begin{tabular}{lc}
\hline Fracture site & Total number \\
\hline Para-symphseal fractures & 14 \\
Body fractures & 7 \\
Angle fractures & 4 \\
Condyle fractures & 3 \\
Sub-condylar fractures & 3 \\
Zygomatico-maxillary complex fractures & 6 \\
\hline
\end{tabular}

The modes of trauma in this study varied from road traffic accidents, fall from a height and interpersonal assault. The leading cause was road traffic accidents accounting for $70 \%$ of the twenty cases presented in the study.

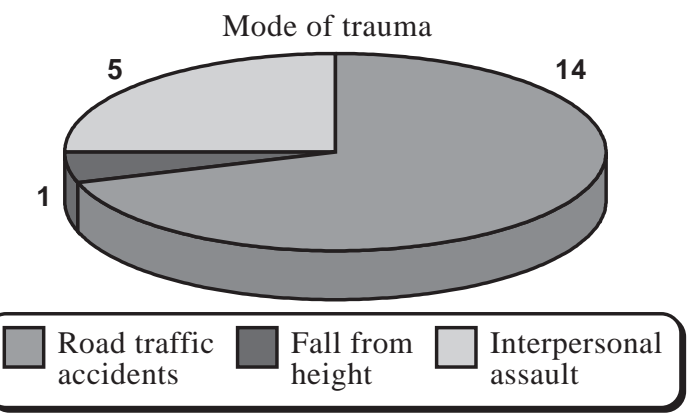

Fig. (3): Showing the modes of trauma in the study.

All patients have been assessed clinically and radiologically pre-operative and post-operative. In the twenty cases included in the study 19 patients presented with malocclusion showing both anterior and posterior and step in bite, only 1 patient has unilateral sub-condylar fracture with normal occlusion. All patients presented in the study have returned to their normal bite before being maloccluded.

Fig. (4): Types and number of open bites in the study.

\begin{tabular}{ll}
\hline Type of open bite & No. \\
\hline Pre-operative anterior open bite & 19 \\
Pre-operative posterior open bite & 17 \\
Pre-operative step in bite & 9 \\
Post-operative malocclusions & None \\
\hline
\end{tabular}


Results of the rating scale questions survey: After collecting the data of all the surveys from all patients, it showed that:

- Convenience during application and removal of the brackets varied from 6 to 9 with an average 7.1 .
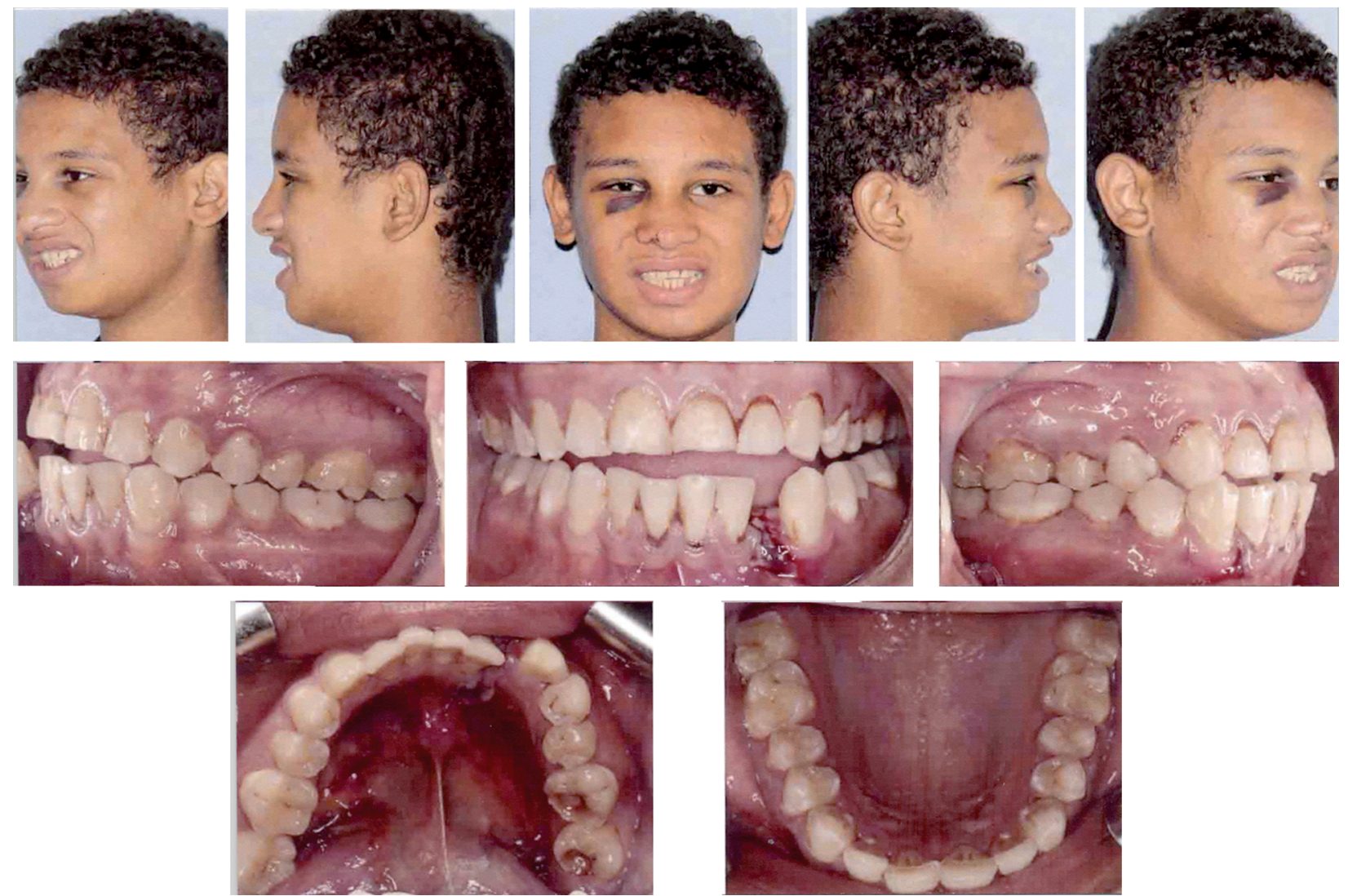

Fig. (5A): A male patient, 15 years old, presented by a left para-symphyseal fracture.

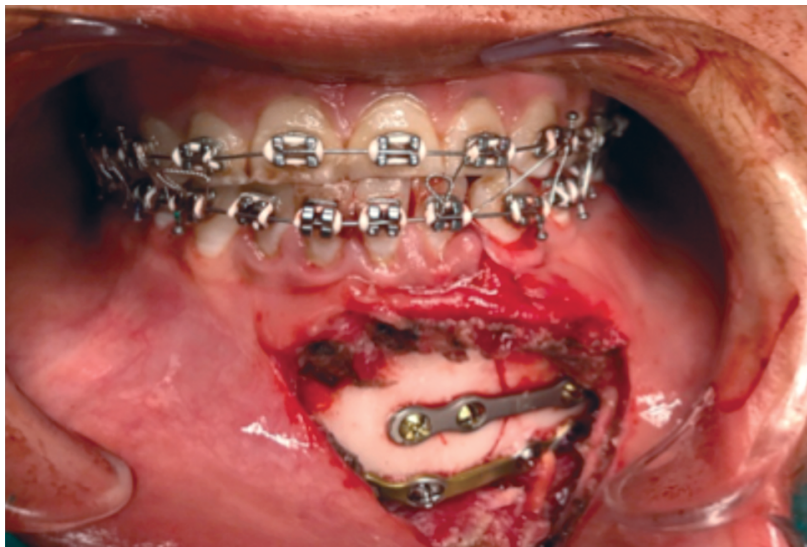

Fig. (5B): Showing intra-operative fixation of the fracture with 2 mini-plates.
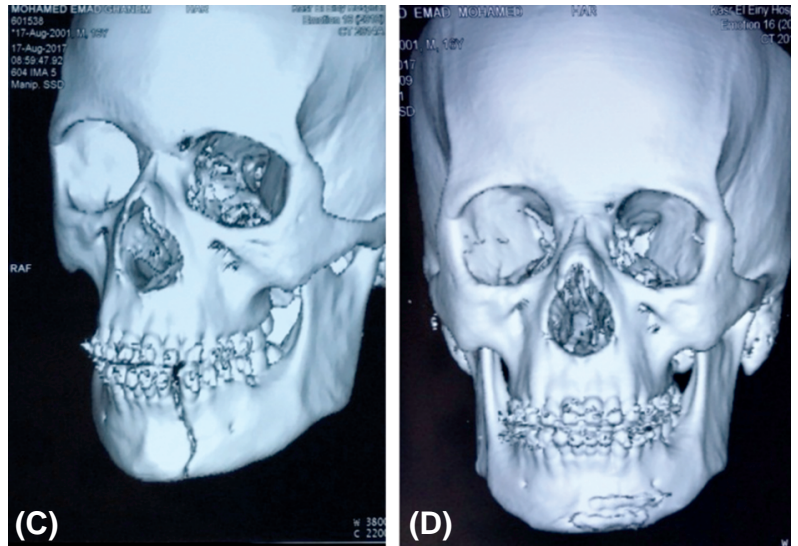

Fig. (5C,D): Showing pre-operative and post-operative CT scan for the same patient. 

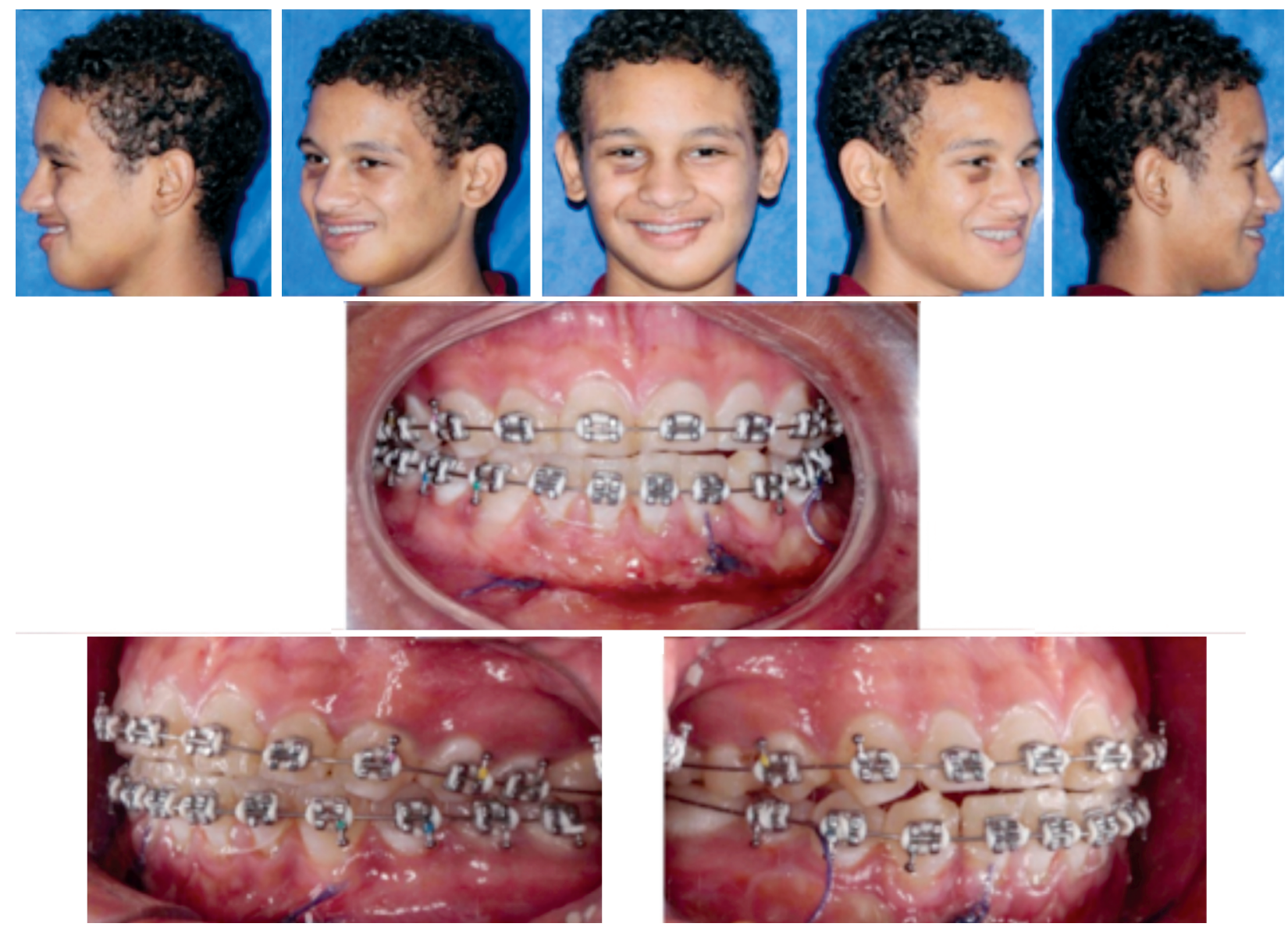

Fig. (5E): Showing the same patient two weeks post-operatively during the period of the orthodontic brackets.
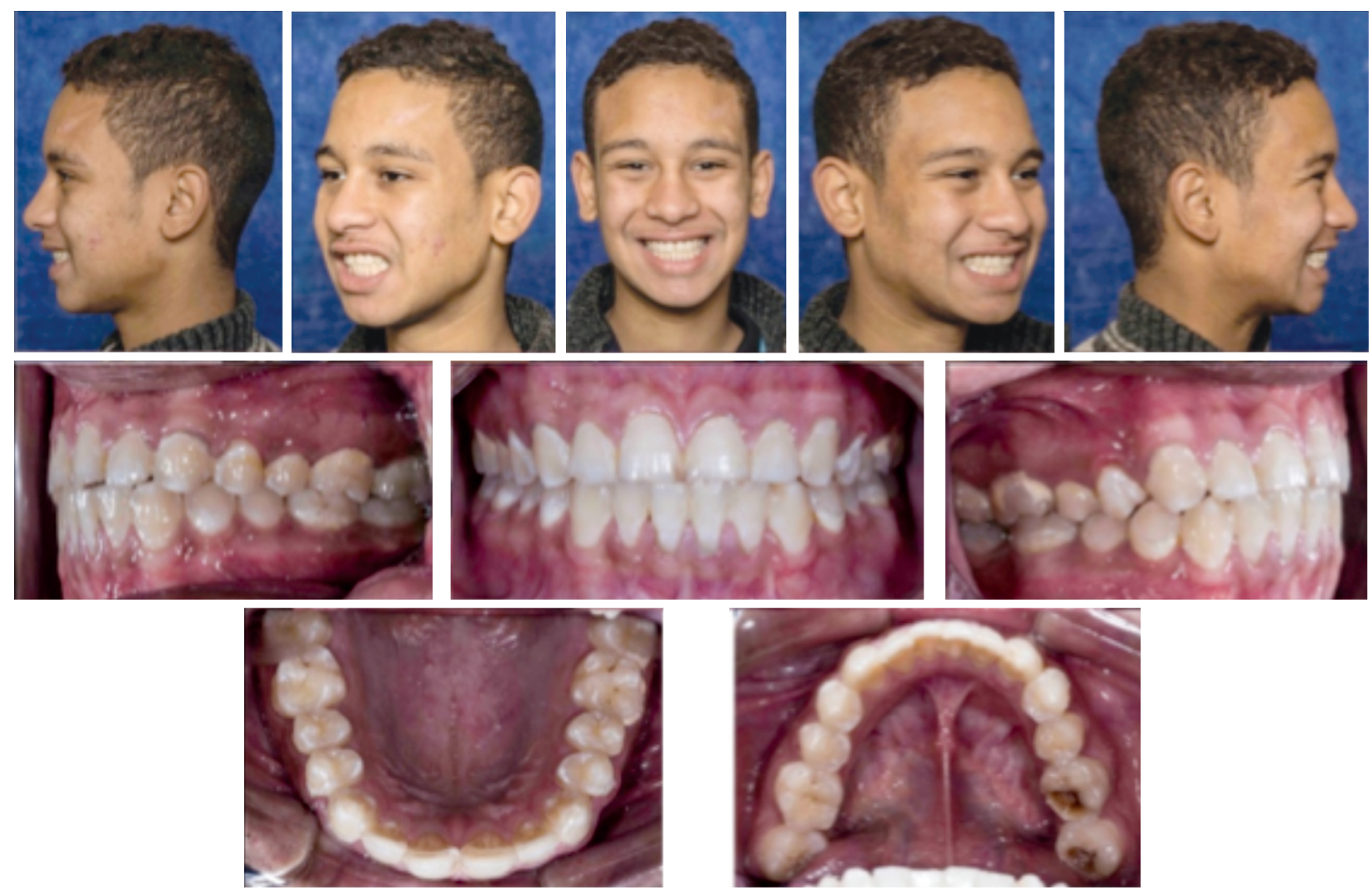

Fig. (5F): Showing the same patient 3 months post-operatively after removal of the orthodontic brackets. 

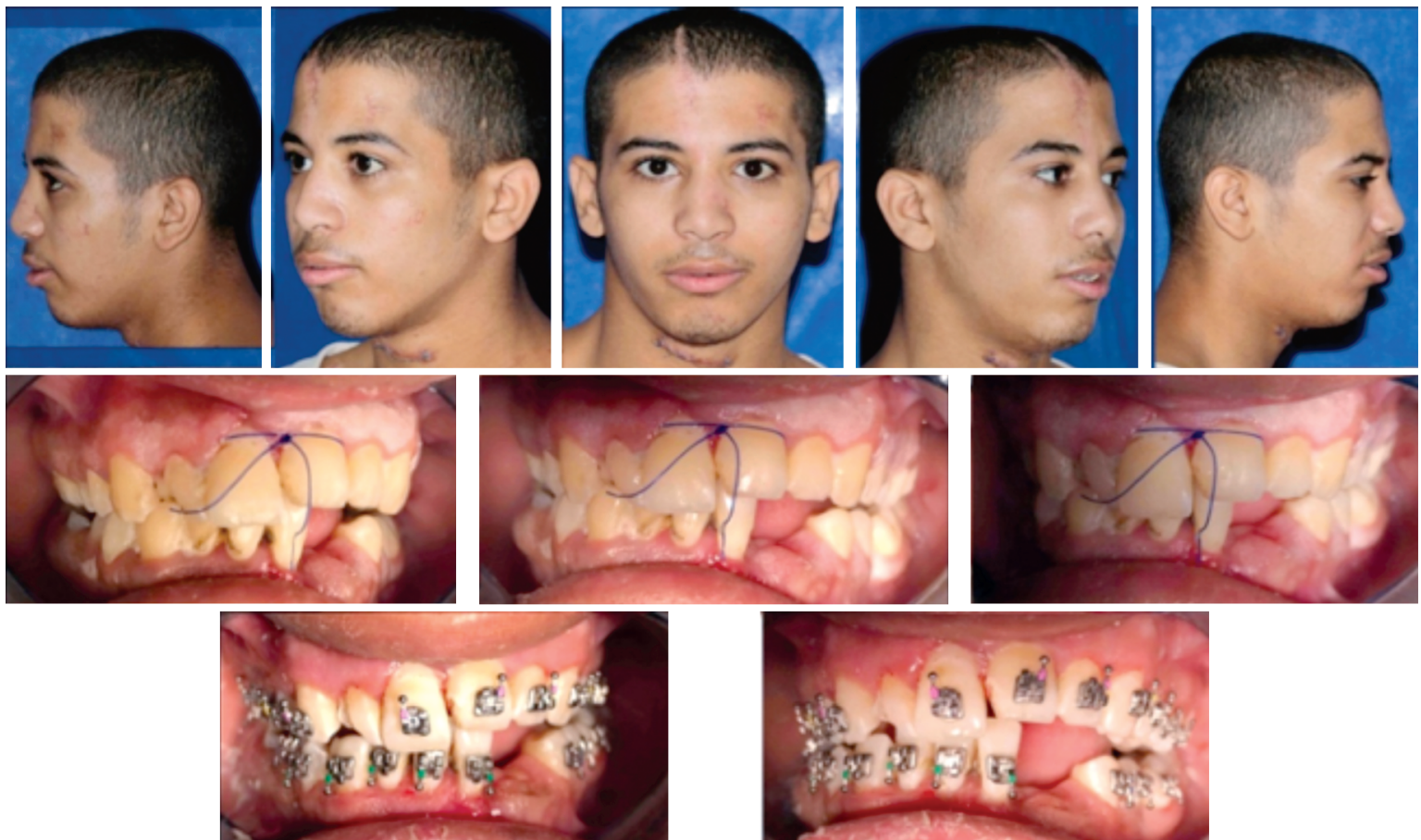

Fig. (6A): A male patient, 19 years old presented by left side Le-fort 1 and midline dento-alveolar maxillary fracture. Patient underwent MMF only using orthodontic appliance with no ORIF.
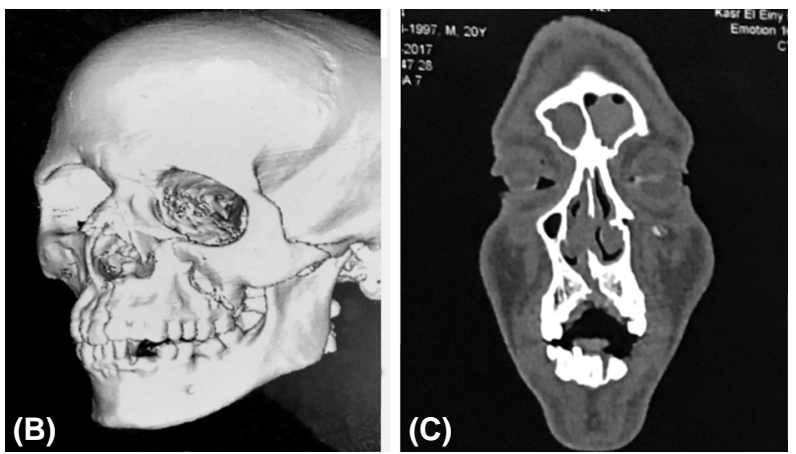

Fig. $(6 \mathrm{~B}, \mathrm{C})$ : Showing CT scan of the same patients before application of the orthodontic appliance.
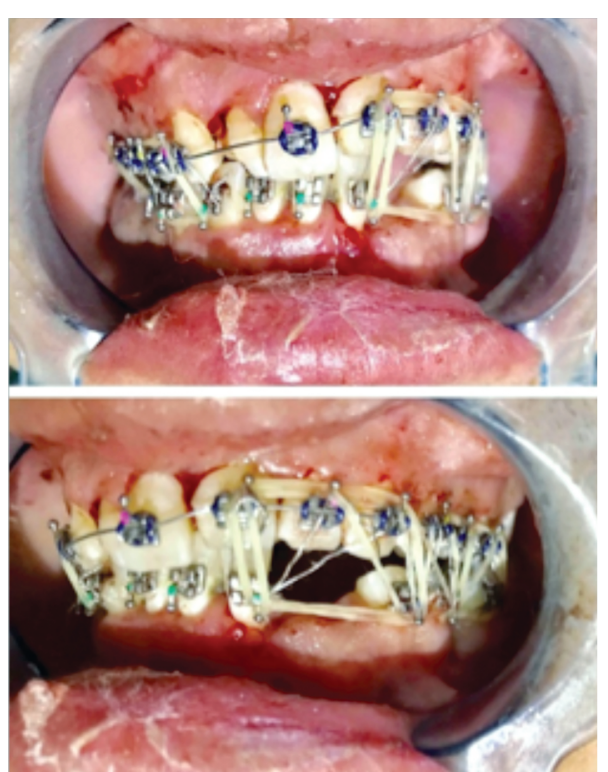

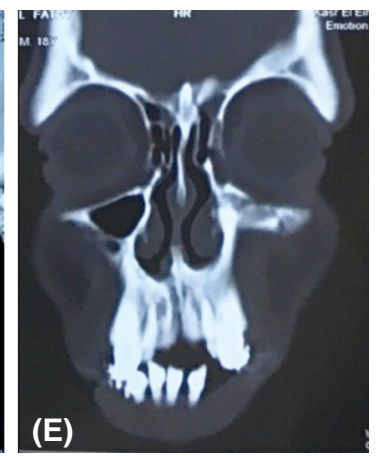

Fig. (6D,E): Showing CT scan of the same patients after application of the orthodontic appliance.
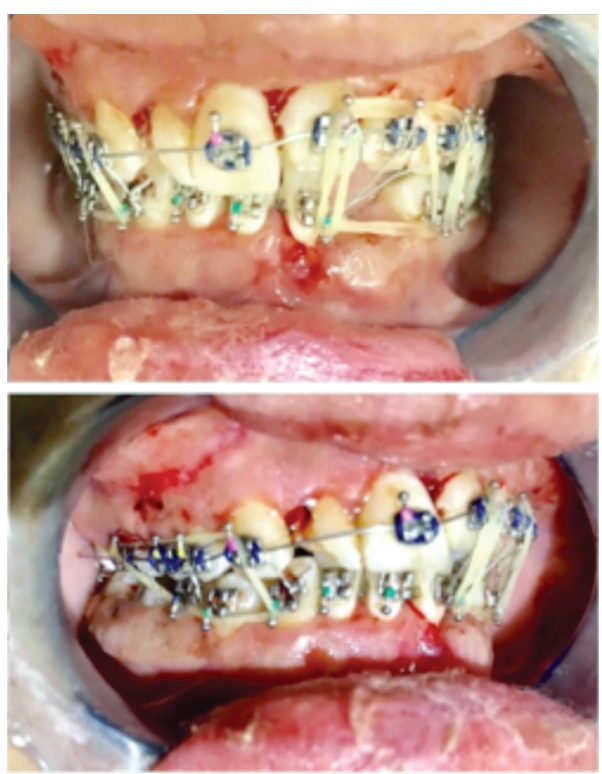

Fig. (6F): Showing a the same patient during the application of the orthodontic brackets that continued for 5 weeks. 

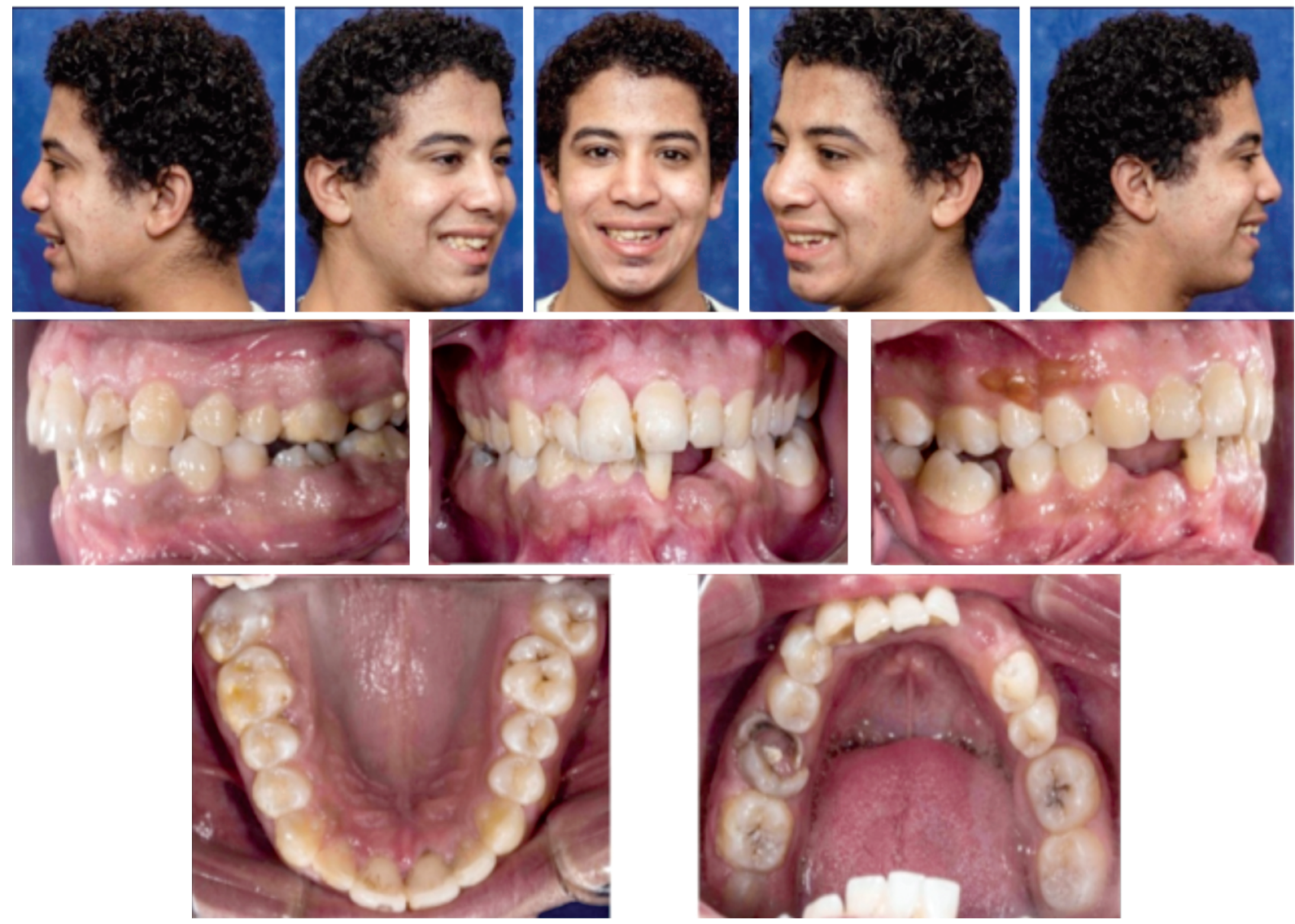

Fig. (6G): Showing the same patients after three weeks from the removal of the orthodontic brackets.
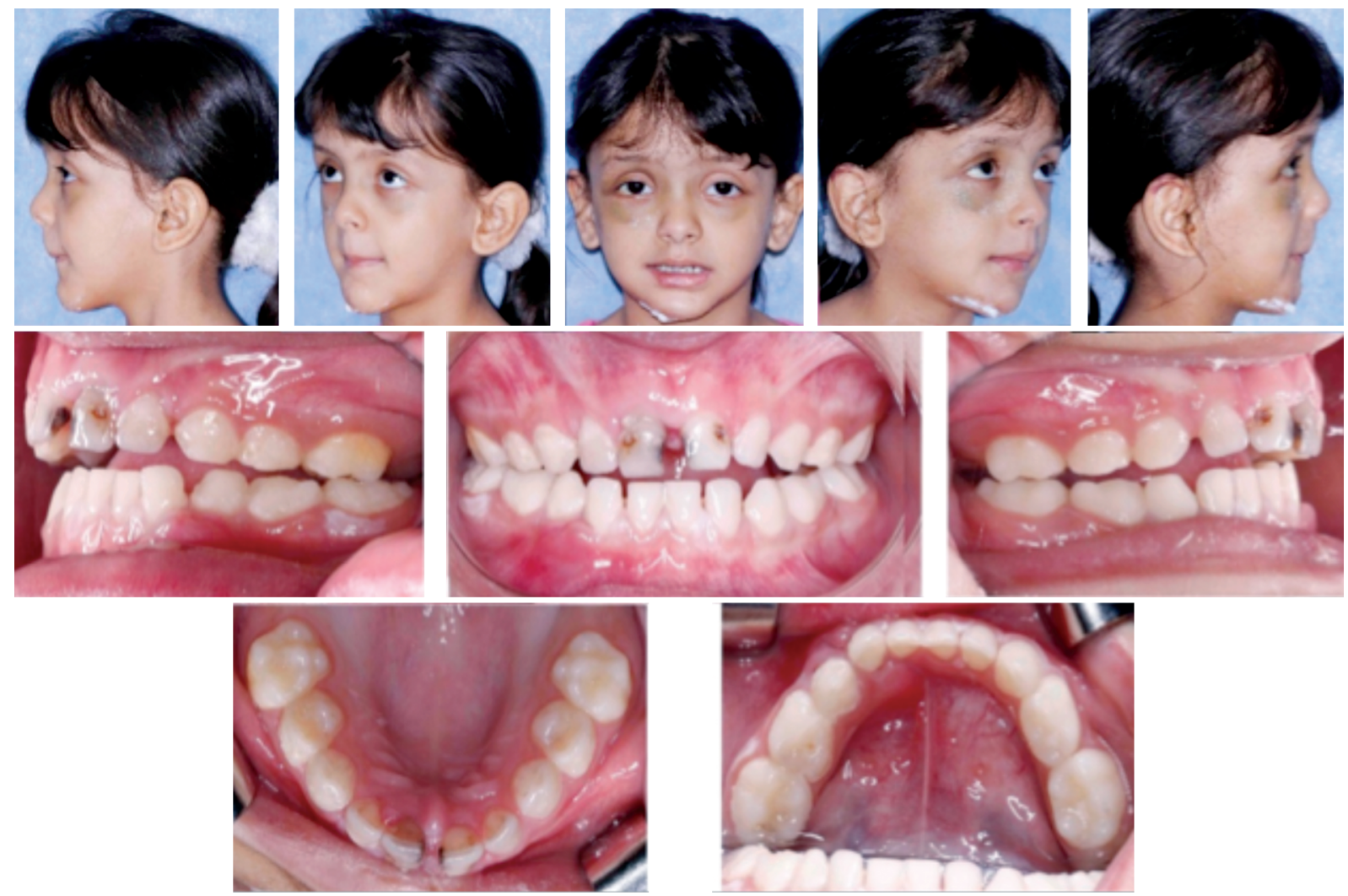

Fig. (7A): A 6 year old female patient presented by right zygomatico-maxillary, right condylar and right para-symphseal fractures post fall from height. Patient underwent MMF only using orthodontic appliance. 

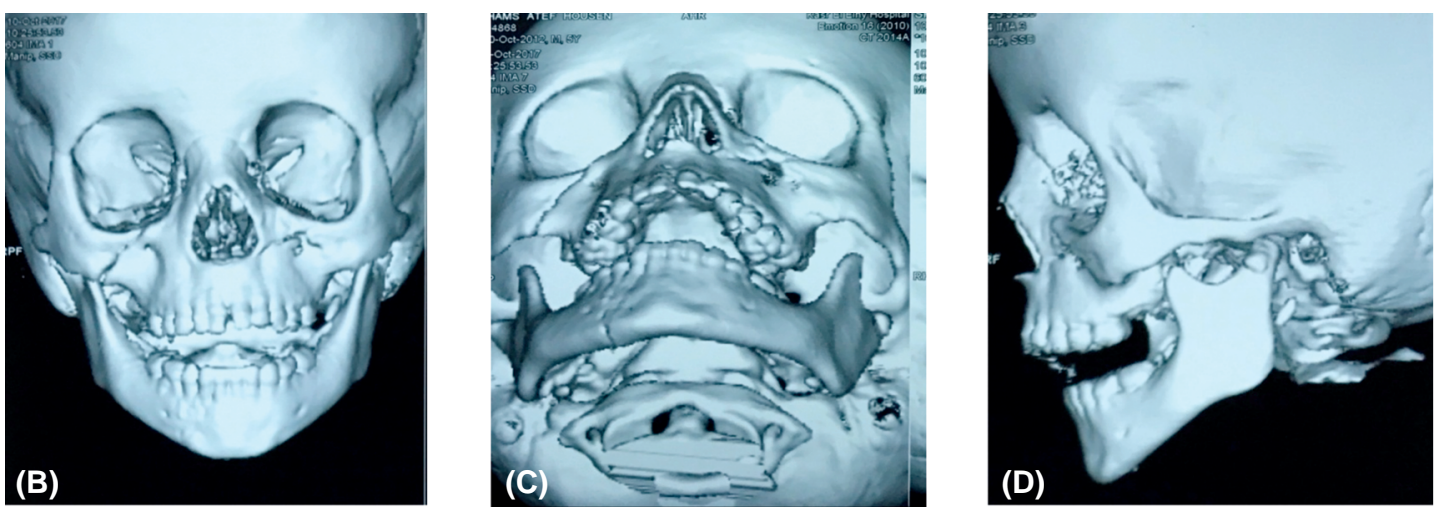

Fig. (7B,C,D): Showing CT scan of the same patients before application of the orthodontic appliance.
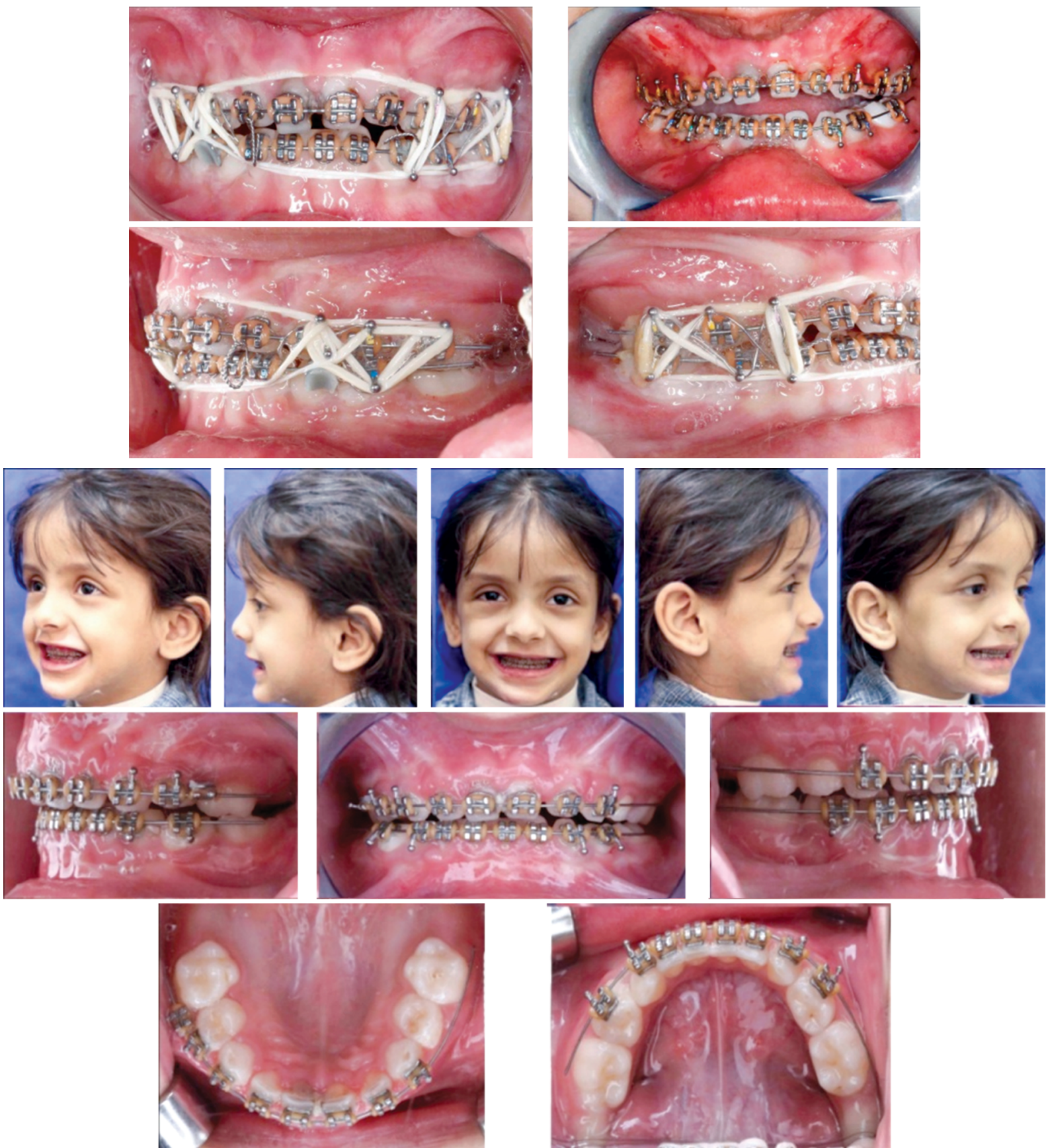

Fig. (7E,F): Showing the same patient during and immediately after the application of the orthodontic brackets immediately after removal of the occlusion that remained for 3 weeks. 

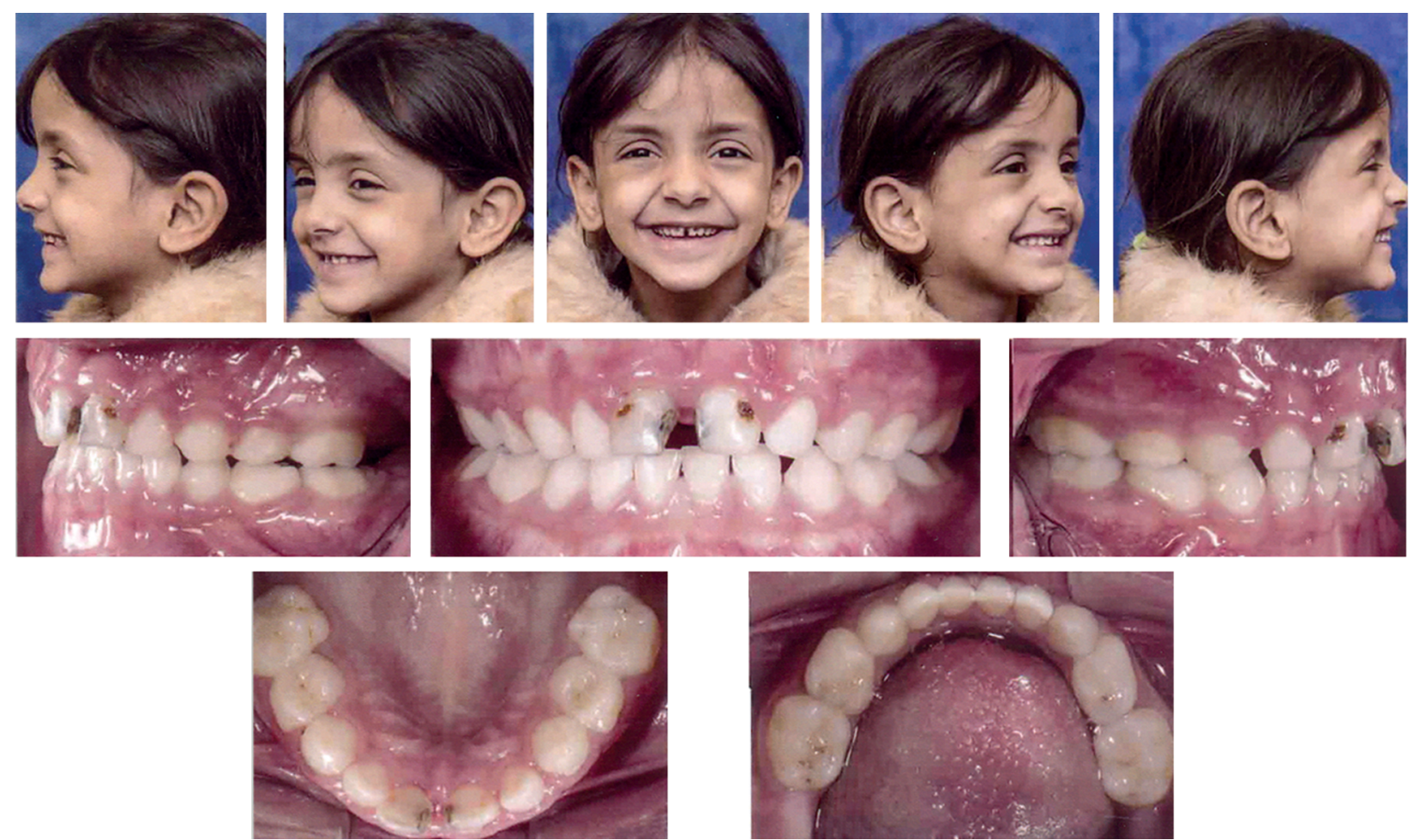

Fig. (7G): Showing the same patient after removal of the orthodontic appliance that remained for 6 weeks.

\section{DISCUSSION}

Maxillo-facial fractures are a common presentation that can be isolated or in combination with other injuries in multi-trauma patients. There adequate management should include both proper precise assessment and decision making to reach the most functional and aesthetic results for the patients with the least early and late complication and inconvenience to those patients [6,7].

Reduction of fractures can be achieved either by closed or open methods. Proper reduction means good alignment of fracture ends, but the ultimate goal is to reach the pre-trauma occlusion that needs proper reliable immobilization to insure the maintenance of this reduction to avoid any postreduction mal-healing and mal-occlusion [8].

There are a lot of methods used to achieve immobilization as using MMF screws, arch bars, interdentally wiring and the modernly era of orthodontic appliance [9]. Simple mandibular fractures can be immobilized using MMF alone if there is adequate number of teeth and if the fracture on the tooth bearing part. Healing can be achieved within 4 weeks and MMF can be done without anesthesia. A short period of MMF with Guiding elastics or wires may be used to complete direct fixation [10].

Orthodontists have a long history of clinical use of an adhesive material to bond brackets to teeth through a technique used by itching the enamel surface of the tooth to make micro irregularities on the tooth surface. The role of the bond is adherence of the adhesive material over the irregularities, after application of brackets elastic traction or wires can be attached. This method has advantages as it can be used without anesthesia, no need for wide mouth opening during application, no gingival or mucosal injury, application and removal can be done without anesthesia, better oral hygiene and patient convenience, costs for the hospital and patient reduced by reducing the rate of patient admission and the need of general anesthesia [11].

One of the early studies to use orthodontic appliance was the study done by Roland et al., treated only patients in whom normal maxillarymandibular occlusal relationships could be achieved without strong inter-maxillary traction. Intermaxillary fixation was maintained for 3 to 6 weeks, oral hygiene was good and patient acceptance was excellent. No damage to the teeth has resulted from the use of this technique [5].

Another study was done by Magennis \& Craven when they reported the orthodontic brackets where traction was done using elastics in the treatment of only 3 cases of fracture mandible and he reported that this technique has proved a useful adjunct to other methods available for treating fractured mandibles [11]. 
Utley et al., made a study on 32 patients with mandibular fractures and orthodontic direct bonded bracket fixation and they stated that they can serve as a single treatment method in patients with favorable, less complicated mandibular fracture with satisfactory results [12].

Multiple case reports were done for the use of direct bonded brackets as the one done by Kaya et al., who reported the use of DBB and ORIF in a single case of parasymphyseal fracture in an adult male, another one done by Madhusudhan where he used direct bonded brackets and ORIF in a pediatric patient in right sub-condylar fracture, another case report done by Prasad et al., where they reported the use of direct bonded brackets in a case presented with multiple facial fractures (symphysis, left sub-condylar and left zygomatic fractures), the MMF using orthodontic bracket was done alone without ORIF. All these studies expanded and widened the range of inter-maxillary fixation using orthodontic bracket as an effective alternative method to other MMF methods [13,14].

A retrospective study done by RIA et al., stated that all mandibular fractures treated only with MMF in the past 12 month were identified. The length of admission, time to theatre and time in theatre was identified. Costing for this was performed and the amount that potentially could have been saved were calculated. They stated that in suitable cases orthodontic brackets and elastics can be used as an effective method of MMF. This can reduce the hospital admission rate and need for general anesthesia. This will not only reduce the cost for the hospital but also the cost for the patient [15].

In this study, it is a prospective study done on 20 cases presented with maxillo-mandibular fractures at Kasr Al-Ainy, Cairo University Hospital Emergency Plastic Surgery Department. They were all managed by orthodontic appliance. Throughout the study the benefits of orthodontic appliance were revealed, and with every fracture fixed we got more accustomed to the technique's difficulties and challenges, gaining more knowledge and experience in the technique application that helped us to venture into a more complex fractures or fracture site and malocclusion. When compared to other techniques used in MMF of maxillofacial fractures, orthodontic appliance has proven to us multiple advantages over traditional MMF systems.

In this study results, the effectiveness of MMF for immobilization using orthodontic brackets to achieve a pre-trauma occlusion were assessed both clinically and radiologically for a post-operative period of 6 months despite the variety of fractures. Occlusions were acceptable with no significant post-operative mal-occlusion with either radiologically or clinically in the anterior and posterior bites.

One of the main benefits of applying orthodontic brackets is the ability to use it as MMF without anesthesia and this can reduce the operative time if ORIF is indicated after the MMF was done as the application of the orthodontic brackets can be done bed side pre-operatively. It can also reduce the hospital admission rate and the need of general anesthesia if the patient was prepared to do MMF only without ORIF. This cannot only reduce costs for the hospital but also for the patient, patient acceptance and comfort is noticed and oral hygiene is facilitated.

All patients underwent orthodontic brackets application bed side except two patient where the application was done intra-operative due to the presence of other concomitant injuries that interfered with that application of the orthodontic brackets bedside.

The convenience of the patients and the surgeons was noticed. A survey with rating scale questions was given to all patients of the Study after removal the orthodontic brackets in which the patients had to give a rate from 1-10 for items and we found that there was an excellent convenience during oral feeding and oral hygiene and during the time of application and removal of the orthodontic brackets with almost no presence of mucosal injuries and pain during this period of application. No surgeon injuries were detected during orthodontic brackets application in the contrary to the use of erich arch bars which is commonly and widely used by maxilla-facial surgeons.

\section{Conclusion:}

Maxillofacial fractures are a common presentation in Plastic Surgery Department emergencies, but with the continuous efforts of scientific researchers that has affected the evolution of treatment, it has offered the treating surgeon the luxury to choose the best suitable management plan and best method of MMF. With the peak age affected between 5 and 30 years old as shown in this study, it necessitates choosing the management plan of the least morbidity offering the fixation that withstands the test of time of the lives ahead of them.

Orthodontic brackets technique's stability allowed using it as a maxillary mandibular fixation 
even alone without open reduction and internal fixation, thus it is an effective method for maxillarymandibular fixation.

Orthodontic brackets technique has proven to cause a significant impact on returning the patients to their pre-traumatic bite. This makes it able to be used instead of erich arch bar in maxillofacial fracture as a method of maxillary-mandibular fixation.

\section{Ethical statement:}

- Funding:

The authors did not receive any funding for the completion of this work.

\section{- Declaration of conflicting interests:}

The authors declared no potential conflicts of interest with respect to the research, authorship, and/or publication of this article.

\section{- Ethical committee approval:}

Plastic Surgery Department, Cairo University Ethical Committee approval was obtained prior to the study.

\section{- Informed consent:}

Informed consent was obtained from all patients enrolled in the study. The study included five minors in whom informed consent was taken acquired from their legal guardians. The consent included details of the pre-operative photographing, the operative procedure and the post-operative sequels treatment. Patients or their legal guardians (in the five cases) approved enrolment in the study and publication.

\section{REFERENCES}

1- Raymond D.E.F., Fonseca J., Dexter H. and Barber P.: Powers, Oral and Maxillofacial Trauma, 4th Edition, Fourth. Linda Duncan, 2013.
2- Franz Härle M.C. and Bill C.T.: Atlas of Craniomaxillofacial Osteosynthesis: Microplates, Miniplates, and Screws, 2009.

3- Hardt N. and Kuttenberger J.: Craniofacial trauma: Diagnosis and management, 2010.

4- Michael Perry P.B. and Andrew B.: Fractures of the Facial Skeleton, Vol. 1, 2015.

5- Minami R.T., Morrill L.R. and Weber J.: "Intermaxillary fixation with orthodontic brackets bonded to teeth," Plast. Reconstr. Surg., Vol. 54, No. 4, pp. 492-4, 1974.

6- Malik N.A.: Textbook of Oral and Maxillofacial Surgery, $2^{\text {nd }}$ ed. jitendar, 2008.

7- Michael Miloro L.J.P.: Peterson's Principles of Oral And Maxillofacial Surgery. Shelton, CT: People's Medical Pub. House-USA, 2012.

8- WY H. and SJ M.: "Rigid internal fixation vs. traditional techniques for the treatment of mandible fractures.," in Journal of trauma, Vol. 30, No. 8, pp. 1032-6, 1990.

9- Janis E.: Essentials of Plastic Surgery, Second Edition $2^{\text {nd }}$ Edition, 2nd ed. Ohio State University, 2014.

10- Peter W. Booth B. and Eppley R.S.: Maxillofacial Trauma and Esthetic Facial Reconstruction, Second, 2011.

11- Magennis P. and Craven P.: "Modification of orthodontic brackets for use in intermaxillary fixation," Br. J. Oral Maxillofac. Surg., Vol. 28, No. 2, pp. 136-7, 1990.

12- Utley D., Utley J., Koch R. and Goode R.: "Direct Bonded Orthodontic Brackets for Maxillomandibular Fixation.," Laryngoscope, Vol. 108, No. 9. pp. 1338-45, 1998.

13- Demet Kaya C., et al.: "Use Of Acrylic Occlusal Splint And Direct Bonded Brackets For Intermaxillary Fixation In The Treatment Of Unilateral Parasymphyseal And Condylar Fractures: A Case Report," Clin. Dent. Res., Vol. 37, No. 1, pp. 46-50, 2013.

14- Sivaprasad L.P., Anila K.K., Ranjith K.P. and Krishnakumar K.: "Intermaxillary Fixation With Orthodontic Bracketsa Case,” Eur. J. Pharm. Med. Res., Vol. 4, No. 7, pp. 61821, 2017.

15- Ria S., Bakir A., Perry M., Ria B., Attwall R. and Balasundaram I.: "The financial case for using orthodontic brackets as a method of intermaxillary fixation in suitable mandible fractures,” Int. J. Oral Maxillofac. Surg., Vol. 46, p. 264, 2017. 